\title{
Computer-Aided Simulation of Gas Adsorption Processes in Nanopores
}

\author{
A. V. Korchuganov ${ }^{1, \text { a) }}$, K. P. Zolnikov ${ }^{1,2}$, D. S. Kryzhevich ${ }^{1,2}$, \\ Yu. V. Grinyaev ${ }^{1}$, and S. G. Psakhie ${ }^{1,3,4}$ \\ ${ }^{1}$ Institute of Strength Physics and Materials Science SB RAS, Tomsk, 634055, Russia \\ ${ }^{2}$ National Research Tomsk State University, Tomsk, 634050, Russia \\ ${ }^{3}$ National Research Tomsk Polytechnic University, Tomsk, 634050, Russia \\ ${ }^{4}$ Skolkovo Institute of Science and Technology, Skolkovo, 143025, Russia \\ a) Corresponding author: avkor@ispms.tsc.ru
}

\begin{abstract}
A computer-aided simulation of the behavior of molecular gas-phase hydrogen in spherical nanopores was accomplished. The calculations were performed within the frame of molecular dynamics. Interaction between hydrogen molecules is described on the basis of adaptive intermolecular reactive empirical bond-order (AIREBO) potential. The interaction of gas with the walls of nanopores was calculated using the Lennard-Jones potential. The paper also contains the calculated distributions of gas density and velocities of molecules for different temperatures and pressures. The paper states that the adsorption properties of gas systems in nanopores can be described by varying the parameters of the potential of interaction with pore walls.
\end{abstract}

Keywords: nanoporous materials, adsorption, molecular dynamics

\section{INTRODUCTION}

The adsorption phenomenon serves as a basis for numerous technical solutions and establishes the area for state of art research works [1-6]. Among the diversity of practical applications of adsorption phenomena one can mention the purification and separation of substances, and gas chromatography [7,8]. Usage of nanoporous adsorbents as promising storage of hydrogen is yet another vital application of the aforementioned phenomena [9]. The foregoing considerations stimulate the active study of adsorption phenomena on the surface of various materials and nanostructures. The adsorption in porous materials is dramatically affected by porosity, dimensions and shape of pores [10]. The positive adsorption requires satisfactory wettability of the material surface. It is noteworthy that the methods used for determining the amount of absorbed substance are widely used for estimating the geometrical parameters of nanoporous materials, volume and dimensions of pores. Understanding of the peculiarities of adsorption phenomena of gases in nanopores requires the simulation at atomic scale, which allows describing the properties and behavior of nanosystems of various origins [11-13].

The aim of the present work is computer-aided simulation of adsorption dynamics of molecular hydrogen in spherical nanopores and impact of interaction parameters in the "gas-nanopore wall" system on the process of adsorption. Herein, the wall properties will be defined by specifying corresponding parameters of the interaction potential of hydrogen molecule with pore surface.

\section{FORMAL DESCRIPTION}

The object of the study is two-atom $\mathrm{H}_{2}$ gas residing in spherical nanopores. The simulation of gas behavior was realized on the basis of the method of molecular dynamics with the use of LAMMPS computational software [14]. The atomic interaction was described on the basis of adaptive intermolecular reactive empirical bond-order

International Conference on Physical Mesomechanics of Multilevel Systems 2014

AIP Conf. Proc. 1623, 299-302 (2014); doi: 10.1063/1.4898941

(C) 2014 AIP Publishing LLC 978-0-7354-1260-6/\$30.00 
(AIREBO) potential [15]. This potential enables satisfactory simulation of both chemical reactions and molecular interaction of hydrocarbon systems in gas, liquid and solid phases. It was derived on the basis of the reactive empirical bond-order potential of Brenner [16].

The diameter of spherical pores that served for the study of gaseous hydrogen behavior varied from 12.5 to $50 \mathrm{~nm}$. The interaction of hydrogen molecules with the walls of pores was described by the Lennard-Jones potential.

$$
F=4 \varepsilon\left(\left(\frac{\sigma}{r}\right)^{12}-\left(\frac{\sigma}{r}\right)^{6}\right),
$$

where $\varepsilon, \sigma$ are constants, $r$ is the distance between atom and wall. The calculations were carried out for the initial temperature of $300 \mathrm{~K}$ and gas densities ranging from $4.5 \times 10^{-05} \mathrm{~g} / \mathrm{cm}^{3}$ to $3.6 \times 10^{-04} \mathrm{~g} / \mathrm{cm}^{3}$ (the density in normal conditions is $\left.8.99 \times 10^{-05} \mathrm{~g} / \mathrm{cm}^{3}\right)$. The initial coordinates of molecules in a pore were set by a random number generator; the simulated system was evolving for $500 \mathrm{~ns}$ up to reaching the equilibrium state. During the evolution radial distributions of gas density and temperature were being estimated and averaged. The temperature was estimated as follows:

$$
T=\frac{\sum_{i}^{N} m_{i} v_{i} 2}{3 k},
$$

where $N$ is the number of molecules in the simulated system, $k$ is Boltzmann's constant, $m_{i}, v_{i}$ are the mass and velocity of the $i$-th molecule, respectively. The pressure of the simulated gas was calculated using the following expression:

$$
P=\frac{N k T}{V}
$$

where $V$ is the volume of a pore.

\section{RESULTS AND DISCUSSION}

The calculation results demonstrate that until the system reaches the equilibrium state, the potential energy rises, while the gas temperature and pressure monotonically decrease (Fig. 1). Such behavior of the simulated gas is connected with adsorption processes in the area of nanopore wall. Such effect is clearly seen in Fig. 2, which demonstrates in polar coordinates the spatial distribution of molecules in a pore at different moments of time. If at the initial moment of time the gas molecules are uniformly distributed in pore volume (Fig. 2(a)), then on the further evolution of the system the concentration of molecules in near-wall area (its boundaries are highlighted in the Figure by the dashed lines) starts to rise (Fig. 2(b)), until it reaches the equilibrium value (Fig. 2(c)).

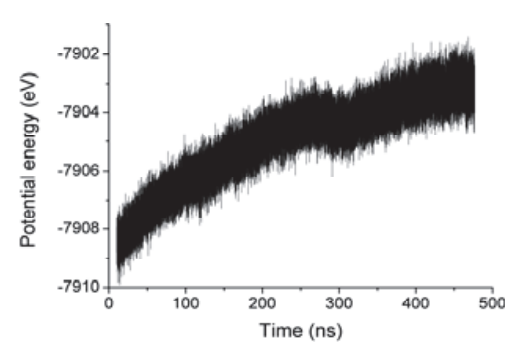

(a)

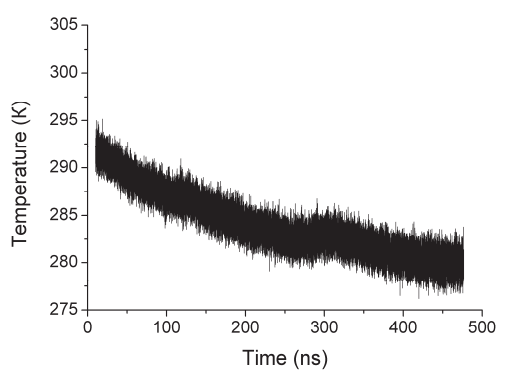

(b)

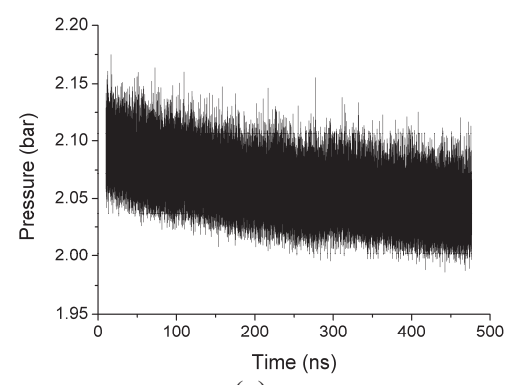

(c)

FIGURE 1. Dependence of various parameters on time in a pore with the diameter of $50 \mathrm{~nm}$ :

(a) energy, (b) temperature and (c) gas pressure 


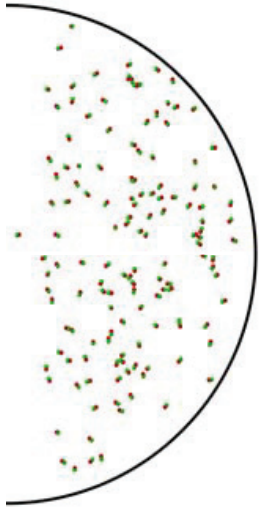

(a)

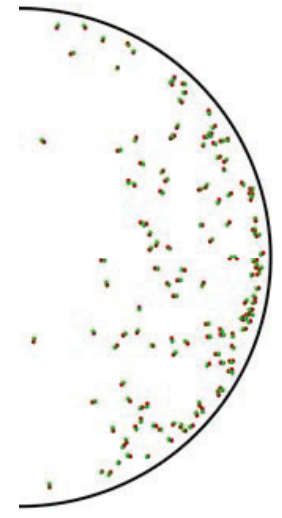

(b)

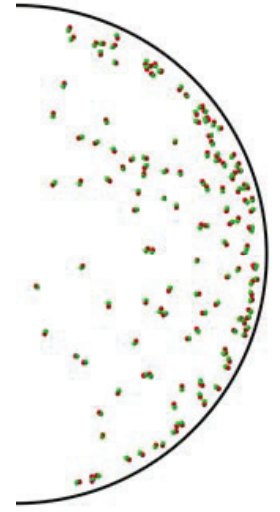

(c)

FIGURE 2. Distribution of molecules in polar coordinates at different moments of time:

(a) $0.05 \mathrm{~ns}$, (b) $250 \mathrm{~ns}$ and (c) $500 \mathrm{~ns}$. Pore diameter is $12.5 \mathrm{~nm}$

The quantitative variations of the radial distribution of gas density in pores with different diameter are depicted in Fig. 3(a). To estimate the density variation the volume of a pore was divided into spherical layers with the thickness of $1 \AA$. After reaching the state close to equilibrium, the gas density was reckoned using the averaged data obtained in the representative time interval $(50 \mathrm{~ns})$. Figure 3(a) evidences that the gas density in near-wall area is approximately two times larger than that in the volume of a pore. Concurrently, the peak position on the derived curve corresponds to the minimum potential of interaction between the wall and a hydrogen atom.

Adsorption characteristics of a material are largely dependent on the potential of interaction between a pore wall and gas molecules. Changing the potential parameters (1) one can alter the degree of gas adsorption in a pore. For instance, Figure 3(b) demonstrates the radial distribution of the gas density for various values of potential parameter $\varepsilon$, which defines the potential well depth. Note that, the more is the value of $\varepsilon$ (the more the potential well depth), the higher is the gas density in wall area.

We also analyzed the radial distribution of velocities of hydrogen molecules. The calculations elucidated that the molecule velocities tend to decrease from the pore center to its wall (Fig. 4). To some extent it is due to the effect of adsorption of hydrogen molecules having lesser velocities. The magnitude of this effect will be determined by the gas temperature and pressure, as well as the parameters of "pore wall-gas" interaction, in particular, the potential well depth.

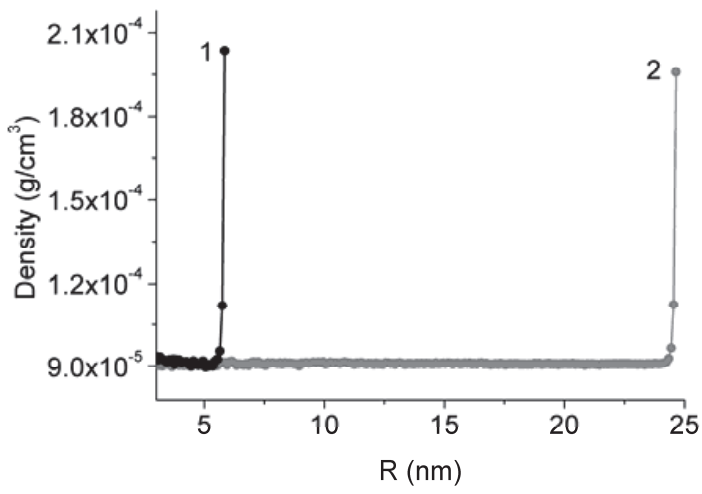

(a)

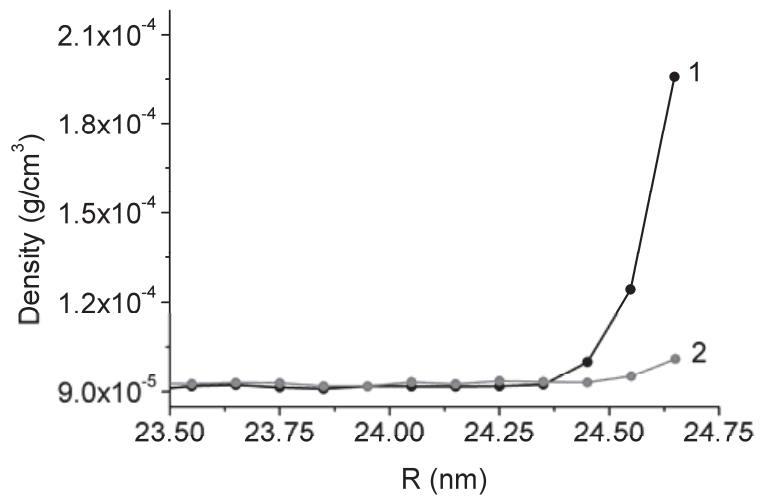

(b)

FIGURE 3. Radial distribution of gas density: (a) in pores with different diameter, curve 1 corresponds to estimations for the pore of $12.5 \mathrm{~nm}$ in diameter, curve 2 corresponds to $50 \mathrm{~nm}$ pore; (b) in a $50 \mathrm{~nm}$ pore for various values of $\varepsilon=0.01(1), 0.002 \mathrm{eV}(2)$ 


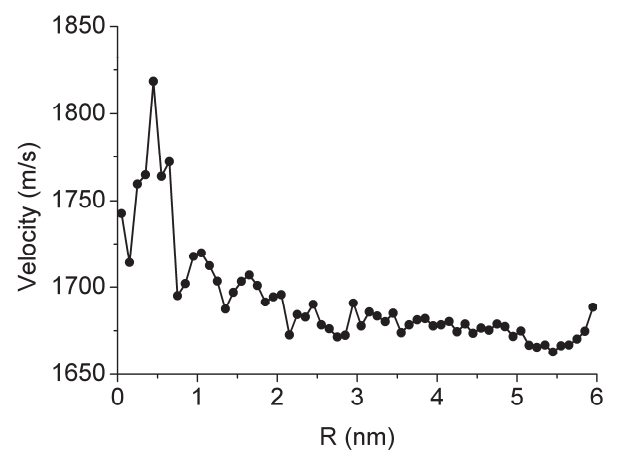

FIGURE 4. Radial distribution of gas molecule velocities in a pore with the diameter of $12.5 \mathrm{~nm}$

\section{ACKNOWLEDGMENTS}

The studies were performed with the state financial support provided by the Ministry of Education and Science of the Russian Federation (project identifier: RFMEFI57814X0002) and the Program for Fundamental Research of State Academies of Sciences for 2013-2020.

\section{REFERENCES}

1. A. C. Dillon, K. M. Jones, T. A. Bekkedahl, et al., Nature 386, 377 (1997).

2. W.-Q. Deng, X. Xu, and W. A. Goddard, Phys. Rev. Lett. 92, 166103 (2004).

3. J. L. C. Rowsell and O. M. Yaghi, J. Am. Chem. Soc. 128, 1304 (2006).

4. W.-C. Xu, K. Takahashi, Y. Matsuo, Y. Hattori, M. Kumagai, S. Ishiyama, K. Kaneko, and S. Iijima, Int. J. Hydrogen Energy 32, 2504 (2007).

5. Y. Yürüm, A. Taralp, and T. Veziroglu, Int. J. Hydrogen Energy 34, 3784 (2009).

6. T. Mitsui, M. K. Rose, E. Fomin, D. F. Ogletree, and M. Salmeron, Lett. Nature 422, 705 (2003).

7. T. Lu, E. M. Goldfield, and S. K. Gray, J. Phys. Chem. B 110(4), 1742-51 (2006).

8. J. Hodgkinson and R. P. Tatam, Meas. Sci. Technol. 24, 012004 (2013).

9. L. Schlapbach and A. Zuttel, Nature 414(6861), 353-8 (2001).

10. I. Krkljus, T. Steriotis, G. Charalambopoulou, A. Gotzias, and M. Hirscher, Carbon 57, 239 (2013).

11. S. G. Psakh'e and K. P. Zol'nikov, Tech. Phys. Lett. 23, 555 (1997).

12. A. I. Dmitriev, K. P. Zolnikov, S. G. Psakhie, et al., Theor. Appl. Fract. Mech. 43, 324 (2005).

13. S. G. Psakh'e, K. P. Zol'nikov, and D. Y. Saraev, Tech. Phys. Lett. 24, 99 (1998).

14. S. J. Plimton, J. Comp. Phys. 117, 1 (1995).

15. S. J. Stuart, A. B. Tutein, and J. A. Harrison, J. Chem. Phys. 112, 6472 (2000).

16. D. W Brenner, Phys. Rev. B 42, 9458 (1990). 